\title{
Optimal location of a thin rigid inclusion for a problem describing equilibrium of a composite Timoshenko plate with a crack
}

\author{
Nyurgun Lazarev ${ }^{1,2^{*}}$, Natalyya Romanova ${ }^{1}$ and Galina Semenova ${ }^{1}$
}

"Correspondence: nyurgun@ngs.ru ${ }^{1}$ North-Eastern Federal University, Yakutsk, Russian Federation

${ }^{2}$ Lavrentyev Institute of

Hydrodynamics SB RAS, Novosibirsk, Russian Federation

\begin{abstract}
We consider equilibrium problems for a cracked composite plate with a thin cylindrical rigid inclusion. Deformation of an elastic matrix is described by the Timoshenko model. The plate is assumed to have a through crack that does not touch the rigid inclusion. In order to describe mutual nonpenetration of the crack faces we impose a boundary condition in the form of inequality on the crack curve. For a family of appropriate variational problems, we analyze the dependence of their solutions on the location of the rigid inclusion. We formulate an optimal control problem with a cost functional defined by an arbitrary continuous functional on the solution space, while the location parameter of inclusion is chosen as the control parameter. The existence of a solution to the optimal control problem and a continuous dependence of the solutions in a suitable Sobolev space with respect to the location parameter are proved.
\end{abstract}

Keywords: Variational inequality; Optimal control problem; Nonpenetration; Nonlinear boundary conditions; Crack; Rigid inclusion

\section{Introduction}

Mathematical models and methods related to analysis of the mechanical properties and behavior of composite materials are sought to provide optimal engineering properties of composites [1-31]. The presence of heterogeneity in the form of microcracks, cracks, and inclusions in loaded structures, can lead to significant local stresses, which in turn, can cause their destruction. In this regard, the issues of justification and examination of mathematical models describing the mechanical influence of defects on stress-strain state of composites are very important.

From a mathematical point of view, the study of composite solids with cracks are often associated with significant difficulties due to the presence of irregular components of boundaries. While studying the problems of the theory of cracks, it is appropriate to take into account the possibility of contact mechanical interaction between the crack faces, which, in particular, leads to the need to use of boundary conditions of the Signorini type. These conditions have the form of inequalities and describe the nonpenetration of the opposite faces of the crack. Nonlinearity of such problems of the theory of cracks leads to

(c) The Author(s) 2020. This article is licensed under a Creative Commons Attribution 4.0 International License, which permits use sharing, adaptation, distribution and reproduction in any medium or format, as long as you give appropriate credit to the original author(s) and the source, provide a link to the Creative Commons licence, and indicate if changes were made. The images or other third party material in this article are included in the article's Creative Commons licence, unless indicated otherwise in a credit line to the material. If material is not included in the article's Creative Commons licence and your intended use is not permitted by statutory regulation or exceeds the permitted use, you will need to obtain permission directly from the copyright holder. To view a copy of this licence, visit http://creativecommons.org/licenses/by/4.0/. 
the use of variational formulations. This variational approach in studying the nonlinear problems of the theory of cracks began to be actively elaborated since the 1990s [32-35]. Among the studies in this field let us note the recently developed nonlinear models describing the equilibrium state of composite solids having cracks on the interfacial boundary of inclusions. For the mentioned models, the carrier matrix is assumed to be elastic, whereas the inclusion is either absolutely rigid (for instance, see [8-10,13-18, 20, 23]) or elastic and described by other constitutive relations (in this case, we have the so-called junction problems [26-31]). The optimal control problems for the shape of the bodies with with cracks and rigid inclusions are studied, for example, in [10-12, 15, 17, 18, 21, 22]. Employing the shape-topological sensitivity analysis [36], the existence of a solution of an optimal control problem related to the best choice of the location and shape of small inclusions was shown in [37]. The existence of an optimal location of a thin rigid inclusion for a two-dimensional model describing equilibrium of a cracked body is proved in [11].

In this article, under study is a model which describes equilibrium state of a composite plate consisting of an elastic carrier matrix, a thin rigid cylindrical inclusion, and a crack at a certain distance from the inclusion. The plate is supposed to be fixed on the external boundary, whereas, on the crack curve, the nonpenetration conditions on the crack faces are imposed. In the framework of this model, we consider a family of problems depending on a parameter $t \in[0, T]$. Each value of the parameter $t$ corresponds to one certain location of a thin rigid inclusion of fixed shape. The optimal control problem analyzed in this paper consists in the best choice of location parameter $t$. A cost functional is defined using an arbitrary continuous functional in the suitable Sobolev space. As our main result, the existence of a solution to the optimal control problem is proved.

\section{A family of equilibrium problems}

Let $\Omega \subset \mathbb{R}^{2}$ be a bounded domain with a smooth boundary $\Gamma \in C^{0,1}$. Suppose that a smooth curve $\gamma \subset \Omega$ without self-intersections has the following properties: $\bar{\gamma} \subset \Omega$, it can be extended to some curve $\Sigma$ dividing $\Omega$ into two subdomains $\Omega_{1}$ and $\Omega_{2}$ with Lipschitz boundaries $\partial \Omega_{1}$ and $\partial \Omega_{2}$ where meas $\left(\Gamma \cap \partial \Omega_{i}\right)>0, i=1,2$. This condition is sufficient for Korn's inequality to hold in the non-Lipschitz domain $\Omega_{\gamma}=\Omega \backslash \bar{\gamma}$. Let $\left(0, x_{2}^{0}\right)$ be the point which is the left end of the curve $\Xi, \Xi \subset \Omega_{\gamma}$, defined as the graph of the function $g(t) \in C^{1}[0, T]$ (see Fig. 1). Let us also consider the family of curves $\beta_{t}, t \in[0, T]$ defined by the relations

$$
\beta_{t}=\{x+(t, g(t)) \mid x \in \hat{\beta}\}, \quad t \in C[0, T]
$$

where $\hat{\beta}=\left\{\left(x_{1}, x_{2}\right) \mid x_{2}=\lambda\left(x_{1}\right),-a \leq x_{1} \leq 0\right\}, \lambda \in C^{1}[-a, 0], 0<\lambda^{\prime}(t), \lambda(0)=0$.

For the mentioned geometrical objects we assume that the following assumption is fulfilled.

Assumption The curve $\gamma$ can be extended to a simple closed curve $\Sigma$ that bounds a domain $O, \bar{O} \subset \Omega$. In addition,

(1) we have the inclusion $\beta_{0}^{T} \subset O$;

(2) the boundaries of the domains $\Omega \backslash \bar{O}$ and $O \backslash \beta_{\theta}^{\mu}$ are Lipschitz for all $\mu$ and $\theta$ such that $0 \leq \theta<\mu \leq T$. The sets $\beta_{0}^{T}$ and $\beta_{\theta}^{\mu}$ are defined by the relations

$$
\beta_{\theta}^{\mu}=\bigcup_{t \in[\theta, \mu]} \beta_{t}, \quad 0 \leq \theta<\mu \leq T .
$$


Figure 1 Geometry of the problem

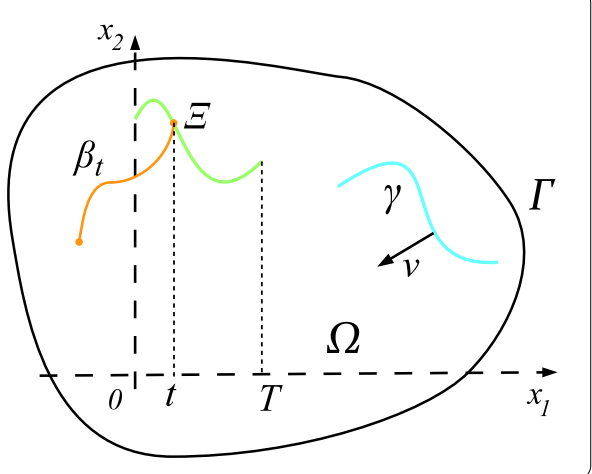

We define a three-dimensional Cartesian space $\left\{x_{1}, x_{2}, z\right\}$ such that the set $\left\{\Omega_{\gamma}\right\} \times\{0\} \subset$ $\mathbb{R}^{3}$ corresponds to the middle plane of the plate. We assume that the thickness $2 h$ of the plate is constant and is equal to two, i.e. $h=1$. The curve $\gamma$ defines a crack (a cut) in the plate. This means that the cylindrical surface of the through crack specified by the relations $x=\left(x_{1}, x_{2}\right) \in \gamma,-1 \leq z \leq 1$ where $|z|$ is the distance to the middle plane. For a fixed parameter $t \in[0, T]$ we suppose that the thin rigid inclusion is specified by the set $\beta_{t} \times[-1 ; 1]$. The elastic part of the plate corresponds to the domain $\Omega_{\gamma} \backslash \bar{\beta}_{t}$. Depending on the direction of the normal $v=\left(v_{1}, v_{2}\right)$ to $\gamma$ we will speak about a positive face $\gamma^{+}$or a negative face $\gamma^{-}$of the curve $\gamma$. The jump [ $\left.v\right]$ of the function $v$ on the curve $\gamma$ is found by the formula $[v]=\left.v\right|_{\gamma^{+}}-\left.v\right|_{\gamma^{-}}$.

Denote by $(W, w)$ the vector of mid-plane displacements $\left(x \in \Omega_{\gamma}\right)$, where $W=\left(w_{1}, w_{2}\right)$ are the displacements in the plane and $\left\{x_{1}, x_{2}\right\}$ and $w$ are the displacements along the axis $z$. We denote the angles of rotation of a normal fiber by $\psi=\psi(x)=\left(\psi_{1}, \psi_{2}\right)\left(x \in \Omega_{\gamma}\right)$.

Introduce the tensors describing the deformation of the transversely isotropic plate

$$
\varepsilon_{i j}(\psi)=\frac{1}{2}\left(\psi_{i, j}+\psi_{j, i}\right), \quad \varepsilon_{i j}(W)=\frac{1}{2}\left(w_{i, j}+w_{j, i}\right), \quad i, j=1,2\left(v_{, i}=\frac{\partial v}{\partial x_{i}}\right) .
$$

The tensors of moments $m(\psi)=\left\{m_{i j}(\psi)\right\}$ and stresses $\sigma(W)=\left\{\sigma_{i j}(W)\right\}$ are expressed by the formulas (summation is performed over repeated indices) [38]

$$
m_{i j}(\psi)=a_{i j k l} \varepsilon_{k l}(\psi), \quad \sigma_{i j}(W)=3 a_{i j k l} \varepsilon_{k l}(W), \quad i, j, k, l=1,2
$$

where the nonzero components of elasticity tensor $A=\left\{a_{i j k l}\right\}$ are as follows:

$$
a_{i i i i}=D, \quad a_{i i j j}=D \kappa, \quad a_{i j i j}=a_{i j i j}=D(1-\kappa) / 2, \quad i \neq j, i, j=1,2,
$$

where $D$ and $\kappa$ are the constants: $D$ is a cylindrical rigidity of the plate, $\kappa$ is the Poisson ratio, $0<\kappa<1 / 2$. The transverse forces in the Timoshenko-type model are specified by the expressions

$$
q_{i}(w, \psi)=\Lambda\left(w, i+\psi_{i}\right), \quad i=1,2,
$$

where $\Lambda=2 k^{\prime} s^{\prime} h, k^{\prime}$ is the shear coefficient, $s^{\prime}$ is the shear modulus in areas perpendicular to the middle plane of the plate, and $\Lambda$ is the constant [38]. Let $B(G, \cdot, \cdot)$ be a bilinear form 
defined by the equality

$$
B(G, \chi, \bar{\chi})=\int_{G}\left\{m_{i j}(\psi) \varepsilon_{i j}(\bar{\psi})+\Lambda\left(w_{i}+\psi_{i}\right)\left(\bar{v}_{, i}+\bar{\psi}_{i}\right)+\sigma_{i j}(W) \varepsilon_{i j}(\bar{W})\right\} d x,
$$

with some subdomain $G \subset \Omega_{\gamma}, \chi=(W, w, \psi), \bar{\chi}=(\bar{W}, \bar{w}, \bar{\psi})$. The potential energy functional of the plate occupying the region $\Omega_{\gamma}$ has the form

$$
\Pi(\chi)=\frac{1}{2} B\left(\Omega_{\gamma}, \chi, \chi\right)-\int_{\Omega_{\gamma}} F \chi d x, \quad \chi=(W, w, \psi)
$$

where $F=\left(f_{1}, f_{2}, f_{3}, \mu_{1}, \mu_{2}\right) \in L^{2}\left(\Omega_{\gamma}\right)^{5}$ is the vector specifying the external loads [38].

Introduce the Sobolev spaces

$$
H^{1,0}\left(\Omega_{\gamma}\right)=\left\{v \in H^{1}\left(\Omega_{\gamma}\right) \mid v=0 \text { a.e. on } \Gamma\right\}, \quad H\left(\Omega_{\gamma}\right)=H^{1,0}\left(\Omega_{\gamma}\right)^{5} .
$$

Due to the presence of a rigid inclusion in the plate, restrictions of the functions describing displacements $(W, w)$ and angles of rotation $\psi$ to the curve $\beta_{t}$ satisfy a special kind of relations. We introduce the space which allows us to characterize the properties of a thin rigid inclusion [19],

$$
R\left(\beta_{t}\right)=\left\{\zeta \mid \zeta(x)=\left(b x_{2}+c_{1},-b x_{1}+c_{2}, a_{0}+a_{1} x_{1}+a_{2} x_{2},-a_{1},-a_{2}\right) ; x \in \beta_{t}\right\},
$$

where $b, c_{1}, c_{2}, a_{0}, a_{1}, a_{2} \in \mathbb{R}$. The condition of mutual nonpenetration of the opposite faces of the crack is given by [20]

$$
[W] v \geq|[\psi] v| \text { on } \gamma
$$

We formulate the contact problem of the plate with a rigid inclusion

$$
\inf _{\chi \in K_{t}} \Pi(\chi)
$$

where

$$
K_{t}=\left\{\chi=(W, w, \psi) \in H\left(\Omega_{\gamma}\right)|[W] v \geq|[\psi] v \mid \text { on } \gamma,\left.\chi\right|_{\beta_{t}} \in R\left(\beta_{t}\right)\right\}
$$

is the set of admissible functions. Note that the inclusion $\chi \in H\left(\Omega_{\gamma}\right)$ assumes that the homogeneous boundary-value conditions hold:

$$
w=0, \quad \psi=W=(0,0) \quad \text { on } \Gamma .
$$

It can be shown that the set $K_{t}$ is convex and closed in the Hilbert space $H\left(\Omega_{\gamma}\right)$ [20]. Due to the estimate

$$
B\left(\Omega_{\gamma}, \chi, \bar{\chi}\right) \leq c_{1}\|\chi\|\|\bar{\chi}\|, \quad\|\cdot\|=\|\cdot\|_{H\left(\Omega_{\gamma}\right)},
$$


where the constant $c_{1}>0$ is independent of $\chi \in H\left(\Omega_{\gamma}\right)$ and $\bar{\chi} \in H\left(\Omega_{\gamma}\right)$, the symmetric bilinear form of $B\left(\Omega_{\gamma}, \chi, \bar{\chi}\right)$ is continuous with respect to $H\left(\Omega_{\gamma}\right)$. The coercivity of the functional $\Pi(\chi)$ follows from the inequality

$$
B\left(\Omega_{\gamma}, \chi, \chi\right) \geq c\|\chi\|^{2}, \quad \forall \chi \in H\left(\Omega_{\gamma}\right)
$$

where the constants $c>0$ is independent of $\chi$ (see [39]).

Remark 1 The inequality (8) yields the equivalence of the standard norm and the seminorm determined by the bilinear form $B\left(\Omega_{\gamma}, \cdot, \cdot\right)$ in the space $H\left(\Omega_{\gamma}\right)$.

The above properties of the energy functional $\Pi(\chi)$, the bilinear form $B\left(\Omega_{\gamma}, \cdot, \cdot\right)$, and the set $K_{t}$ allow one to establish the existence of a unique solution $\xi_{t}=\left(U_{t}, u_{t}, \phi_{t}\right) \in K_{t}$ for problem (6); see [34]. Symmetry and continuity of the bilinear form $B\left(\Omega_{\gamma}, \cdot, \cdot\right)$ and the properties of the set $K_{t}$ provide the equivalence of problem (6) to the variational inequality

$$
\xi_{t} \in K_{t}, \quad B\left(\Omega_{\gamma}, \xi_{t}, \chi-\xi_{t}\right) \geq \int_{\Omega_{\gamma}} F\left(\chi-\xi_{t}\right) d x, \quad \forall \chi=(W, w, \psi) \in K_{t} .
$$

\section{Optimal control problem}

For an arbitrary continuous functional $G(\chi): H\left(\Omega_{\gamma}\right) \rightarrow \mathbb{R}$ we can define the cost functional $J:[0, T] \rightarrow \mathbb{R}$ with the help of the equality $J(t)=G\left(\xi_{t}\right)$, where $\xi_{t}$ is the solution of the problem (6). The mentioned continuity property is fulfilled for many physically motivated functionals, for example, the functional

$$
G_{1}(\chi)=\left\|\chi-\chi_{0}\right\|_{H\left(\Omega_{\gamma}\right)}
$$

characterizes the deviation of the generalized displacement vector from a given function $\chi_{0} \in H\left(\Omega_{\gamma}\right)$.

Consider the optimal control problem:

$$
\text { Find } t^{*} \in[0, T] \text { such that } J\left(t^{*}\right)=\sup _{t \in[0, T]} J(t) \text {. }
$$

Theorem 1 There exists a solution of the optimal control problem (10).

Proof Let $\left\{t_{n}\right\}$ be a maximizing sequence. By the boundedness of the interval $[0, T]$, we can extract a convergent subsequence $\left\{t_{n_{k}}\right\} \subset\left\{t_{n}\right\}$ such that

$$
t_{n_{k}} \rightarrow t^{*} \quad \text { as } k \rightarrow \infty, \quad t^{*} \in[0, T]
$$

Without loss of generality we assume that $t_{n_{k}} \neq t^{*}$ for sufficiently large $k$. Otherwise there would exist a subsequence $\left\{t_{n_{l}}\right\}$ such that $t_{n_{l}} \equiv t^{*}$, and therefore $J\left(t^{*}\right)$ is solution of (10).

Now we take into account Lemma 2 proved below: the solutions $\xi_{k}$ of (6) corresponding to the parameters $t_{n_{k}}$ converge to the solution $\xi_{t^{*}}$ strongly in $H\left(\Omega_{\gamma}\right)$ as $k \rightarrow \infty$. This allows us to obtain the convergence

$$
J\left(t_{n_{k}}\right) \rightarrow J\left(t^{*}\right)
$$


indicating that

$$
J\left(t^{*}\right)=\sup _{t \in[0, T]} J(t) .
$$

The theorem is proved.

\section{Auxiliary lemmas}

Now we have to justify auxiliary lemmas used within the proof of the theorem. In establishing the proof, we needed Lemma 2; however, before proceeding we need first prove the following lemma.

Lemma 1 Let $t^{*} \in[0, t]$ be a fixed number and let $\left\{t_{n}\right\} \subset[0, T]$ be a sequence of numbers converging to $t^{*}$ as $n \rightarrow \infty$. Then for every function $\eta \in K_{t^{*}}$ there are a subsequence $\left\{t_{k}\right\}=$ $\left\{t_{n_{k}}\right\} \subset\left\{t_{n}\right\}$ and a sequence of functions $\left\{\eta_{k}\right\}$ such that $\eta_{k} \in K_{t_{k}}, k \in \mathbb{N}$ and $\eta_{k} \rightarrow \eta$ strongly in $H\left(\Omega_{\gamma}\right)$ as $k \rightarrow \infty$.

Proof Note that if there is a subsequence $\left\{t_{n_{k}}\right\}$ such that $t_{n_{k}}=t$; then the statement of the lemma will hold for $W_{k}=W, k \in \mathbb{N}$. Without loss of generality, we assume that $\left\{t_{n}\right\}$ is a strictly monotonic decreasing sequence such that

$$
t^{*}=\lim _{n \rightarrow \infty} t_{n}
$$

Denote by $\zeta^{*}$ the function describing the structure of $\eta$ on $\beta_{t^{*}}$, i.e.

$$
\zeta^{*}=\eta=\left(b^{*} x_{2}+c_{1}^{*},-b^{*} x_{1}+c_{2}^{*}, a_{0}^{*}+a_{1}^{*} x_{1}+a_{2}^{*} x_{2},-a_{1}^{*},-a_{2}^{*}\right)
$$

on $\beta_{t^{*}}$. We extend the definition of $\zeta^{*}$ to the whole domain $\Omega$ by the equality:

$$
\zeta^{*}(x)=\left(b^{*} x_{2}+c_{1}^{*},-b^{*} x_{1}+c_{2}^{*}, a_{0}^{*}+a_{1}^{*} x_{1}+a_{2}^{*} x_{2},-a_{1}^{*},-a_{2}^{*}\right), \quad x \in \Omega .
$$

By the assumptions, there exists some number $\delta>0$ such that for all $t \in[0, T] \cap\left(t^{*}, t^{*}+\delta\right)$ the set $\beta_{t^{*}}^{t}$ has a Lipschitz boundary. We fix a parameter $t \in[0, T] \cap\left(t^{*}, t^{*}+\delta\right)$ and consider the following family of auxiliary problems:

$$
\text { Find } Q_{t} \in K_{t}^{\prime} \text { such that } p\left(Q_{t}\right)=\inf _{\chi \in K_{t}^{\prime}} p(\chi) \text {, }
$$

where $p(\chi)=B\left(\Omega_{\gamma}, \chi-\eta, \chi-\eta\right)$,

$$
K_{t}^{\prime}=\left\{\chi=(W, w, \psi) \in H\left(\Omega_{\gamma}\right) \mid \chi=\eta \text { on } \gamma^{ \pm},\left.\chi\right|_{\beta_{t^{*}}^{t}}=\zeta^{*}\right\} .
$$

It is easy to see that the functional $p(\chi)$ is coercive and weakly lower semicontinuous on the space $H\left(\Omega_{\gamma}\right)$. One can verify that the set $K_{t}^{\prime}$ is convex and closed in $H\left(\Omega_{\gamma}\right)$. These properties guarantee the existence of a solution $Q_{t}$ of problem (11). Besides, its solution is unique [33]. Since the functional $p(\chi)$ is convex and differentiable on $H\left(\Omega_{\gamma}\right)$, problem (11) can be written in the equivalent form:

$$
Q_{t} \in K_{t}^{\prime}, \quad B\left(\Omega_{\gamma}, Q_{t}-\eta, \chi-Q_{t}\right) \geq 0, \quad \forall \chi \in K_{t}^{\prime} .
$$


Note that $\left\{Q_{t}\right\} \in K_{t}$ for all $t \in[0, T] \cap\left[t^{*}, t^{*}+\delta\right)$. To prove the boundedness of $\left\{Q_{t}\right\}$, we use (12) with an appropriate test function $\hat{\chi}$ that belongs to $K_{t}^{\prime}$ for all $t \in[0, T] \cap$ $\left[t^{*}, t^{*}+\delta\right)$. Such a function $\hat{\chi} \in H\left(\Omega_{\gamma}\right)$ can be constructed by applying a lifting operator for the Lipschitz domains $\Omega \backslash \bar{O}$ and $O \backslash\left(\beta_{0}^{T}\right)$ with the following values on the boundaries: $\hat{\chi}=\zeta^{*}$ on $\partial \beta_{0}^{T}, \hat{\chi}=0$ on $\Gamma, \hat{\chi}=\eta$ on $\gamma^{ \pm}$[33]. The function constructed in the proposed manner obviously belongs to $K_{t}^{\prime}$ for all $t \in[0, T] \cap\left(t^{*}, t^{*}+\delta\right)$, and, hence, it can serve in (12) as a test function. This yields

$$
B\left(\Omega_{\gamma}, Q_{t}-\eta, \hat{\chi}\right)+B\left(\Omega_{\gamma}, \eta, Q_{t}\right) \geq B\left(\Omega_{\gamma}, Q_{t}, Q_{t}\right), \quad \forall t \in[0, T] \cap\left(t^{*}, t^{*}+\delta\right) .
$$

Using the inequality (8) we obtain from this relation the following uniform upper bound:

$$
\left\|Q_{t}\right\| \leq c, \quad \forall t \in[0, T] \cap\left(t^{*}, t^{*}+\delta\right) .
$$

This allows us to extract from the sequence $\left\{Q_{t_{n}}\right\}$ a subsequence $\left\{Q_{l}\right\}, l \in \mathbb{N}$ weakly converging to some function $\widetilde{\eta}$ in $H\left(\Omega_{\gamma}\right)$. Hereinafter, for convenience, we use the following notation for subsequences: $t_{l}=t_{n_{l}}$ and $Q_{l}=Q_{t_{n_{l}}}$.

It is now necessary to show that $\tilde{\eta}=\eta$. By construction we have the inclusion $\left(Q_{l}-\eta\right) \in$ $H_{0}^{1}\left(\Omega_{\gamma} \backslash \bar{\beta}_{t^{*}}\right)^{5}$. Then, taking into account the properties of $H_{0}^{1}\left(\Omega_{\gamma} \backslash \bar{\beta}_{t^{*}}\right)^{5}$, we see that the limit function $(\widetilde{\eta}-\eta)$ belongs to the same functional space.

We consider now the functions of the form $\chi_{l}^{ \pm}=Q_{l} \pm \alpha$, where $\alpha$ is a function defined by zero extension of some arbitrary function $\tilde{\alpha} \in C_{0}^{\infty}\left(\Omega_{\gamma} \backslash \bar{\beta}_{t^{*}}\right)^{5}$ into $\Omega_{\gamma}$. One can note that for sufficiently large $k$ the inclusion $\chi_{l}^{ \pm} \in K_{t_{l}}^{\prime}$ holds. Taking the elements of these sequences, $\left\{\chi_{l}^{+}\right\}$and $\left\{\chi_{l}^{-}\right\}$, as test functions into inequalities (12), we infer

$$
Q_{l} \in K_{t_{l}}^{\prime}, \quad B\left(\Omega_{\gamma}, Q_{l}-\eta, \alpha\right)=0 .
$$

The function $\alpha$ is now fixed and by passing to the limit in (13) it is established that

$$
B\left(\Omega_{\gamma}, \tilde{\eta}-\eta, \alpha\right)=0, \quad \forall \alpha \in C_{0}^{\infty}\left(\Omega_{\gamma} \backslash \bar{\beta}_{t^{*}}\right)^{5} .
$$

Hence, by the density of $C_{0}^{\infty}\left(\Omega_{\gamma} \backslash \bar{\beta}_{t^{*}}\right)$ in $H_{0}^{1}\left(\Omega_{\gamma} \backslash \bar{\beta}_{t^{*}}\right)$, we infer that $\tilde{\eta}-\eta=0$ in $H_{0}^{1}\left(\Omega_{\gamma} \backslash \bar{\beta}_{t^{*}}\right)^{5}$. Consequently, $\tilde{\eta}=\eta$ in $H\left(\Omega_{\gamma}\right)$. Therefore, there is a sequence $\left\{Q_{l}\right\}$ such that $Q_{l} \in K_{t}, l \in \mathbb{N}$ and $Q_{l} \rightarrow \eta$ weakly in $H\left(\Omega_{\gamma}\right)$ as $l \rightarrow \infty$.

Now we are in a position to prove the existence the strongly converging subsequence. We will construct it with the help of $\left\{Q_{l}\right\}$. In fact, by the Mazur theorem, there exist a function $N: \mathbb{N} \rightarrow \mathbb{N}$ and a sequence of the sets of reals $\left\{d(n)_{i} \mid i=n, \ldots, N(n)\right\}$, satisfying $d(n)_{i} \geq 0$ and $\sum_{i=n}^{N(n)} d(n)_{i}=1$, such that the sequence $\left\{\hat{Q}_{n}\right\}$ defined by the convex combination

$$
\hat{Q}_{n}=\sum_{i=n}^{N(n)} \alpha(n)_{i} Q_{i}
$$

converges strongly to $\eta$ in the space $H\left(\Omega_{\gamma}\right)$. Hence, we can define the elements $\eta_{n_{k}}$ of the desired sequence $\eta_{n_{k}}$ by the formulas

$$
\eta_{n_{1}}=\hat{Q}_{N(1)}, \quad \eta_{n_{2}}=\hat{Q}_{N(N(1))}, \quad \ldots \quad \eta_{n_{k}}=\hat{Q}_{N^{k}(1)}, \quad \ldots
$$


Obviously, the elements of the constructed sequence $\left\{\eta_{n_{k}}\right\}$ belong to the corresponding sets $K_{t_{N^{k}(1)}}$ with the parameters $t_{N^{k}(1)}$ which are the elements of the subsequence $\left\{t_{l}\right\} \subset\left\{t_{n}\right\}$. Recalling the assumptions made with respect to $\left\{t_{n}\right\}$ in the beginning of the proof of this lemma, we should note the following: In the contrary case, namely, when the sequence $\left\{t_{n}\right\}$ is strictly monotonically increasing, one has to consider the sets $K_{t}^{\prime}$ defined by the sets of the form $\beta_{t}^{t^{*}}$, where $t \in[0, T] \cap\left(t^{*}-\delta, t^{*}\right)$. Lemma 1 is proved.

Using Lemma 1, we prove the following statement applied in the proof of the theorem.

Lemma 2 Let $t^{*} \in[0, T]$ be a fixed real number. Then $\xi_{t} \rightarrow \xi_{t^{*}}$ strongly in $H\left(\Omega_{\gamma}\right)$ as $t \rightarrow t^{*}$, where $\xi_{t}=\left(U_{t}, u_{t}, \phi_{t}\right), \xi_{t^{*}}=\left(U_{t^{*}}, u_{t^{*}}, \phi_{t^{*}}\right)$ are the solutions of $(6)$, corresponding to parameters $t \in[0, T], t^{*} \in[0, T]$.

Proof We proceed by contradiction. Assume that there exist a number $\epsilon_{0}>0$ and some sequence $\left\{t_{n}\right\} \subset[0, T]$ such that $t_{n} \rightarrow t^{*},\left\|\xi_{n}-\xi_{t^{*}}\right\| \geq \epsilon_{0}$, where the $\xi_{n}=\xi_{t_{n}}, n \in \mathbb{N}$, are the solutions of (6), corresponding to $t_{n}$.

Owing to the inclusion $\chi^{0} \equiv 0 \in K_{t}$ for all $t \in[0, T]$, we can substitute $\chi=\chi^{0}$ in (9) for all $t \in[0, T]$. This leads to

$$
\xi_{t} \in K_{t}, \quad B\left(\Omega_{\gamma}, \xi_{t}, \xi_{t}\right) \leq \int_{\Omega_{\gamma}} F \xi_{t} d x, \quad \forall t \in[0, T]
$$

From this, using (8) we can conclude that for all $t \in[0, T]$ the following uniform estimate holds:

$$
\left\|\xi_{t}\right\| \leq c
$$

with some constant $c>0$ independent of $t$. Consequently, replacing $\left\{\xi_{n}\right\}$ by its subsequence, if necessary, we can assume that $\left\{\xi_{n}\right\}$ converges to some $\tilde{\xi}$ weakly in $H\left(\Omega_{\gamma}\right)$.

Now we show that $\tilde{\xi} \in K_{t^{*}}$. In accordance with the Sobolev embedding theorem [33], we obtain

$$
\begin{aligned}
& \left.\left.\xi_{n}\right|_{\beta_{t^{*}}} \rightarrow \tilde{\xi}\right|_{\beta_{t^{*}}} \quad \text { strongly in } L_{2}\left(\beta_{t^{*}}\right)^{5} \text { as } n \rightarrow \infty, \\
& \left.\left.\xi_{n}\right|_{\gamma} \rightarrow \tilde{\xi}\right|_{\gamma} \quad \text { strongly in } L_{2}(\gamma)^{5} \text { as } n \rightarrow \infty
\end{aligned}
$$

We will use the following well-known property of continuity of traces:

$$
\begin{aligned}
& \left\|\left.v\right|_{\beta_{t^{*}}}-\left.v\left(x_{1}+\left(t_{n}-t^{*}\right), x_{2}+g\left(t_{n}\right)-g\left(t^{*}\right)\right)\right|_{\beta_{t^{*}}}\right\|_{L_{2}\left(\beta_{t^{*}}\right)} \\
& \quad \leq C_{1} \sqrt{\left|t_{n}-t^{*}\right|}\|v\|_{H^{1}\left(\Omega_{\gamma}\right)},
\end{aligned}
$$

where $v \in H^{1}\left(\Omega_{\gamma}\right)$ is an arbitrary function [40]. Note that inequality (17) is based on the boundedness of the derivative of the function $g$. In (17) the traces of the function $v\left(x_{1}+\right.$ $\left.\left(t_{n}-t^{*}\right), x_{2}+g\left(t_{n}\right)-g\left(t^{*}\right)\right)$ on $\beta_{t^{*}}$ are related with the traces of the function $v$ on $\beta_{t_{n}}$ by the equality

$$
\begin{aligned}
& \left.v\left(x_{1}+\left(t_{n}-t^{*}\right), x_{2}+g\left(t_{n}\right)-g\left(t^{*}\right)\right)\right|_{\beta_{t^{*}}}=\left.v\left(y_{1}, y_{2}\right)\right|_{\beta_{t_{n}}}, \\
& \left(y_{1}, y_{2}\right)=\left(x_{1}+\left(t_{n}-t^{*}\right), x_{2}+g\left(t_{n}\right)-g\left(t^{*}\right)\right) .
\end{aligned}
$$


Owing to inequality (17) and estimate (14), we have

$$
\begin{aligned}
& \left\|\left.\xi_{n}\right|_{\beta_{t^{*}}}-\left.\xi_{n}\left(x_{1}+\left(t_{n}-t^{*}\right), x_{2}+g\left(t_{n}\right)-g\left(t^{*}\right)\right)\right|_{\beta_{t^{*}}}\right\|_{L_{2}\left(\beta_{t^{*}}\right)^{5}} \\
& \quad \leq C_{1} \sqrt{\left|t_{n}-t^{*}\right|}\left\|\xi_{n}\right\|_{H\left(\Omega_{\gamma}\right)} \leq C_{2} \sqrt{\left|t_{n}-t^{*}\right|}
\end{aligned}
$$

with some constant $C_{2}>0$ independent of $\xi_{n}$. Since by construction

$$
\begin{aligned}
& \left.\xi_{n}\left(x_{1}+\left(t_{n}-t^{*}\right), x_{2}+g\left(t_{n}\right)-g\left(t^{*}\right)\right)\right|_{\beta_{t^{*}}}=\left.\xi_{n}\right|_{\beta_{t_{n}}}=\zeta_{n} \in R\left(\beta_{t_{n}}\right), \\
& \left.\xi_{n}\left(x_{1}+\left(t_{n}-t^{*}\right), x_{2}+g\left(t_{n}\right)-g\left(t^{*}\right)\right)\right|_{\beta_{t^{*}}} \\
& =\left(b_{n}\left(x_{2}+g\left(t_{n}\right)-g\left(t^{*}\right)\right)+c_{1}^{n},-b_{n}\left(x_{1}+\left(t_{n}-t^{*}\right)\right)+c_{2}^{n},\right. \\
& \left.\quad a_{0}+a_{1}\left(x_{1}+\left(t_{n}-t^{*}\right)\right)+a_{2}\left(x_{2}+g\left(t_{n}\right)-g\left(t^{*}\right)\right),-a_{1},-a_{2}\right)\left.\right|_{\beta_{t^{*}}},
\end{aligned}
$$

passing to the limit in (18) and taking into account (15) together with the relations $t_{n} \rightarrow t^{*}$ and $g\left(t_{n}\right) \rightarrow g\left(t^{*}\right)$ as $n \rightarrow \infty$, we arrive at

$$
\lim _{n \rightarrow \infty} \xi_{n}=\lim _{n \rightarrow \infty}\left(b_{n} x_{2}+c_{1}^{n},-b_{n} x_{1}+c_{2}^{n}, a_{0}^{n}+a_{1}^{n} x_{1}+a_{2}^{n} x_{2},-a_{1}^{n},-a_{2}^{n}\right)=\tilde{\xi}
$$

a.e. on $\beta_{t^{*}}$. In turn, it follows that the numbers $\left\{b_{n}\right\},\left\{c_{i}^{n}\right\},\left\{a_{i}^{n}\right\}, i=1,2$, and $\left\{a_{0}^{n}\right\}$ converge to certain numbers $b^{*}, c_{i}^{*}, a_{i}^{*}, i=1,2, a_{0}^{*}$, respectively. Hence,

$$
\tilde{\xi}(x)=\left(b^{*} x_{2}+c_{1}^{*},-b^{*} x_{1}+c_{2}^{*}, a_{0}^{*}+a_{1}^{*} x_{1}+a_{2}^{*} x_{2},-a_{1}^{*},-a_{2}^{*}\right) \quad \text { a.e. on } \beta_{t^{*}} .
$$

Therefore, $\tilde{\xi} \in R\left(\beta_{t^{*}}\right)$. It remains to show that $\tilde{\xi}$ satisfies the nonpentration inequality (5). In view of (16), we can extract subsequences once again and obtain the following convergence: $\left.\left.\xi_{n}\right|_{\gamma} \rightarrow \tilde{\xi}\right|_{\gamma}$, a.e. on both $\gamma^{+}$and $\gamma^{-}$. Now we pass to the limit in the following inequalities as $n \rightarrow \infty$ :

$$
\left[U_{n}\right] v \geq\left|\left[\phi_{n}\right] v\right| \text { on } \gamma
$$

This leads to $[\tilde{U}] v \geq|[\tilde{\phi} v]|$ on $\gamma$, that is, $\tilde{\xi} \in K_{t^{*}}$.

Our next goals are to prove that $\tilde{\xi}=\xi_{t^{*}}$ and to establish the existence of a sequence $\xi_{n}=\xi_{t_{n}}, n=1,2, \ldots$, of solutions strongly converging to $\xi_{t^{*}}$ in $H\left(\Omega_{\gamma}\right)$. For this purpose, we will pass to a limit in variational inequalities (9) corresponding to various parameters $t$. By Lemma 1, for every $\eta \in K_{t^{*}}$ there exist a subsequence $\left\{t_{k}\right\}=\left\{t_{n_{k}}\right\} \subset\left\{t_{n}\right\}$ and a sequence of functions $\left\{\eta_{k}\right\}$ such that $\eta_{k} \in K_{t_{k}}$ and $\eta_{k} \rightarrow \eta$ strongly on $H\left(\Omega_{\gamma}\right)$ as $k \rightarrow \infty$.

This fact, together with Lemma 2, allows us to pass to the limit as $k \rightarrow \infty$ in the following inequalities obtained from (9) for the values of $t=t_{k}$ and the test functions $\left\{\eta_{k}\right\}$ :

$$
B\left(\Omega_{\gamma}, \xi_{k}, \eta_{k}-\xi_{k}\right) \geq \int_{\Omega_{\gamma}} F\left(\chi_{k}-\xi_{k}\right) d x .
$$

As a result, we deduce

$$
B\left(\Omega_{\gamma}, \tilde{\xi}, \eta-\tilde{\xi}\right) \geq \int_{\Omega_{\gamma}} F(\eta-\tilde{\xi}) d x
$$


for every $\eta \in K_{t^{*}}$. From this we can see that the arbitrariness of $\eta \in K_{t^{*}}$ implies that the last inequality is variational. Therefore, by its unique solvability, we conclude that $\tilde{\xi}=\xi_{t}^{*}$.

It remains to establish the strong convergence of $\xi_{n} \rightarrow \xi_{t^{*}}$. Inserting $\chi=2 \xi_{t}$ and $\chi=0$ into the variational inequalities (9) for $t \in[0, T]$, we infer that

$$
B\left(\Omega_{\gamma}, \xi_{t}, \xi_{t}\right)=\int_{\Omega_{\gamma}} F \xi_{t} d x
$$

for all $t \in[0, T]$. Equality (21) together with the weak convergence $\xi_{n} \rightarrow \xi_{t^{*}}$ in $H\left(\Omega_{\gamma}\right)$ as $n \rightarrow \infty$ implies

$$
\lim _{n \rightarrow \infty} B\left(\Omega_{\gamma}, \xi_{n}, \xi_{n}\right)=\lim _{n \rightarrow \infty} \int_{\Omega_{\gamma}} F \xi_{n} d x=\int_{\Omega_{\gamma}} F \xi_{t^{*}} d x=B\left(\Omega_{\gamma}, \xi_{t^{*}}, \xi_{t^{*}}\right)
$$

Recalling here the equivalence of the norms (see Remark 1), we conclude that $\xi_{n} \rightarrow \xi_{t^{*}}$ strongly in $H\left(\Omega_{\gamma}\right)$ as $n \rightarrow \infty$. Thus, we arrive at a contradiction. Lemma 2 is proved.

\section{Conclusion}

For a family of variational equilibrium problems (9) for composite plates with a thin rigid inclusion and a crack, the study is performed of the dependence of their solutions on the parameter $t \in[0, T]$ characterizing the location of the rigid inclusion.

The existence of the solution to the optimal control problem (10) is proved. For that problem the cost functional $J(t)$ is defined by an arbitrary continuous functional $G$ : $H\left(\Omega_{\gamma}\right) \rightarrow \mathbb{R}$, while the control parameter $t$ specifies location of a thin rigid inclusion. To every value of $t$, there corresponds a curve $\beta_{t}$ defining the rigid inclusion, which is obtained by shifting the curve $\hat{\beta}$ along a given graph $\Xi$.

Acknowledgements

The work was supported by the mega-grant of the Russian Federation Government.

Funding

The first author was supported by the mega-grant of the Russian Federation Government (project no. 14.Y26.31.0013).

Availability of data and materials

Data sharing not applicable to this article as no datasets were generated or analyzed during the current study.

Competing interests

The authors declare that they have no competing interests.

Authors' contributions

All authors contributed equally to the writing of this paper. All authors read and approved the final manuscript.

\section{Publisher's Note}

Springer Nature remains neutral with regard to jurisdictional claims in published maps and institutional affiliations.

Received: 2 October 2019 Accepted: 30 January 2020 Published online: 05 February 2020

References

1. Furtsev, A.I.: On contact between a thin obstacle and a plate containing a thin inclusion. J. Math. Sci. 237(4), 530-545 (2019)

2. Sendeckyj, G.P.: Interaction of cracks with rigid inclusions in longitudinal shear deformation. Int. J. Fract. Mech. 101(1), 45-52 (1974)

3. Dal Corso, F., Bigoni, D., Gei, M.: The stress concentration near a rigid line inclusion in a prestressed, elastic material. Part I. Full-field solution and asymptotics. J. Mech. Phys. Solids 56(3), 815-838 (2019)

4. Kovtunenko, V.A., Leugering, G.: A shape-topological control problem for nonlinear crack-defect interaction: the antiplane variational model. SIAM J. Control Optim. 54(3), 1329-1351 (2016) 
5. Pyatkina, E.V.: Optimal control of the shape of a layer shape in the equilibrium problem of elastic bodies with overlapping domains. J. Appl. Ind. Math. 10(3), 435-443 (2016)

6. Rudoy, E.M., Lazarev, N.P.: Domain decomposition technique for a model of an elastic body reinforced by a Timoshenko's beam. J. Comput. Appl. Math. 334, 18-26 (2018)

7. Annin, B.D., Kovtunenko, V.A., Sadovskii, V.M.: Variational and hemivariational inequalities in mechanics of elastoplastic, granular media, and quasibrittle cracks. Springer Proc. Math. Stat. 121, 49-56 (2015)

8. Khludnev, A., Leugering, G.: On elastic bodies with thin rigid inclusions and cracks. Math. Methods Appl. Sci. 33(16), 1955-1967 (2010)

9. Rudoy, E.M.: Shape derivative of the energy functional in a problem for a thin rigid inclusion in an elastic body. Z. Angew. Math. Phys. 66(4), 1923-1937 (2015)

10. Shcherbakov, V.V.: Shape optimization of rigid inclusions for elastic plates with cracks. Z. Angew. Math. Phys. 67, Article ID 71 (2016). https://doi.org/10.1007/s00033-016-0666-7

11. Lazarev, N.P., Semenova, G.M.: Optimal control of the location of a thin rigid inclusion in the equilibrium problem of an inhomogeneous two-dimensional body with a crack. J. Appl. Ind. Math. 13(1), 2025-2040 (2019)

12. Lazarev, N., Semenova, G.: An optimal size of a rigid thin stiffener reinforcing an elastic two-dimensional body on the outer edge. J. Optim. Theory Appl. 178(2), 614-626 (2018)

13. Neustroeva, N.V.: A rigid inclusion in the contact problem for elastic plates. J. Appl. Ind. Math. 4(4), 526-538 (2010)

14. Lazarev, N.P., Semenova, G.M.: On the connection between two equilibrium problems for cracked bodies in the cases of thin and volume rigid inclusions. Bound. Value Probl. (2019). https://doi.org/10.1186/s13661-019-1201-5

15. Lazarev, N.: Existence of an optimal size of a delaminated rigid inclusion embedded in the Kirchhoff-Love plate. Bound. Value Probl. 2015, Article ID 180 (2015). https://doi.org/10.1186/s13661-015-0437-y

16. Khludnev, A.M., Novotny, A.A., Sokolowski, J., Zochowski, A.: Shape and topology sensitivity analysis for cracks in elastic bodies on boundaries of rigid inclusions. J. Mech. Phys. Solids 57(10), 1718-1732 (2009)

17. Khludnev, A., Negri, A.: Optimal rigid inclusion shapes in elastic bodies with cracks. Z. Angew. Math. Phys. 64(1), 179-191 (2013)

18. Khludnev, A.M.: Shape control of thin rigid inclusions and cracks in elastic bodies. Arch. Appl. Mech. 83(10), 1493-1509 (2013)

19. Lazarev, N., Popova, T., Semenova, G.: Existence of an optimal size of a rigid inclusion for an equilibrium problem of a Timoshenko plate with Signorini-type boundary condition. J. Inequal. Appl. (2016) https://doi.org/10.1186/s13660-015-0954-3

20. Lazarev, N.P., Itou, H., Neustroeva, N.V.: Fictitious domain method for an equilibrium problem of the Timoshenko-type plate with a crack crossing the external boundary at zero angle. Jpn. J. Ind. Appl. Math. 33(1), 63-80 (2016)

21. Lazarev, N.P., Rudoy, E.M.: Optimal size of a rigid thin stiffener reinforcing an elastic plate on the outer edge. Z. Angew. Math. Mech. 97(9), 1120-1127 (2017)

22. Lazarev, N.P., Popova, T.S., Rogerson, G.A.: Optimal control of the radius of a rigid circular inclusion in inhomogeneous two-dimensional bodies with cracks. Z. Angew. Math. Phys. 69(3), Article ID 53 (2018). https://doi.org/10.1007/s00033-018-0949-2

23. Rudoy, E.M., Shcherbakov, V.V.: Domain decomposition method for a membrane with a delaminated thin rigid inclusion. Sib. Èlektron. Mat. Izv. 13(1), 395-410 (2016)

24. Popova, T., Rogerson, G.A.: On the problem of a thin rigid inclusion embedded in a Maxwell material. Z. Angew. Math. Phys. 67, Article ID 105 (2016). https://doi.org/10.1007/s00033-016-0700-9

25. Khludnev, A., Popova, T.: Semirigid inclusions in elastic bodies: mechanical interplay and optimal control. Comput. Math. Appl. 77(1), 253-262 (2019)

26. Faella, L., Khludnev, A.: Junction problem for elastic and rigid inclusions in elastic bodies. Math. Methods Appl. Sci. 39(12), 3381-3390 (2016)

27. Shcherbakov, V.V.: The Griffith formula and J-integral for elastic bodies with Timoshenko inclusions. Z. Angew. Math. Mech. 96(11), 1306-1317 (2016)

28. Khludnev, A.M., Shcherbakov, V.V.: Singular path-independent energy integrals for elastic bodies with Euler-Bernoulli inclusions. Math. Mech. Solids 22(11), 2180-2195 (2017)

29. Khludnev, A.M., Faella, L., Popova, T.S.: Junction problem for rigid and Timoshenko elastic inclusions in elastic bodies. Math. Mech. Solids 22(4), 737-750 (2015)

30. Khludnev, A.M., Popova, T.S.: Junction problem for Euler-Bernoulli and Timoshenko elastic inclusions in elastic bodies. Q. Appl. Math. 74(4), 705-718 (2016)

31. Itou, H., Khludnev, A.M.: On delaminated thin Timoshenko inclusions inside elastic bodies. Math. Methods Appl. Sci. 39(17), 4980-4993 (2016)

32. Khludnev, A.M., Sokolowski, J.: Modelling and Control in Solid Mechanics. Birkhäuser, Basel (1997)

33. Khludnev, A.M., Kovtunenko, V.A.: Analysis of Cracks in Solids. WIT Press, Southampton (2000)

34. Khludnev, A.M.: Elasticity Problems in Nonsmooth Domains. Fizmatlit, Moscow (2010) (in Russian)

35. Khludnev, A.M., Shcherbakov, V.V.: A note on crack propagation paths inside elastic bodies. Appl. Math. Lett. 79(1), 80-84 (2018)

36. Novotny, A.A., Sokolowski, J.: Topological Derivatives in Shape Optimization. Interaction of Mechanics and Mathematics. Springer, Berlin (2013)

37. Leugering, G., Sokolowski, J., Zochowski, A.: Control of crack propagation by shape-topological optimization. Discrete Contin. Dyn. Syst., Ser. A 35(6), 2625-2657 (2015)

38. Pelekh, B.L.: Theory of Shells with Finite Shear Modulus. Nauk Dumka, Kiev (1973)

39. Lazarev, N.P.: An iterative penalty method for a nonlinear problem of equilibrium of a Timoshenko-type plate with a crack. Numer. Anal. Appl. 4(4), 309-318 (2011)

40. Mikhailov, V.P.: Partial Differential Equations. Nauka, Moscow (1976) (in Russian) 